\title{
Lactobacillus species isolated from vaginal secretions of healthy and bacterial vaginosis-intermediate Mexican women: a prospective study
}

\author{
Marcos Daniel Martínez-Peña ${ }^{1,2}$, Graciela Castro-Escarpulli ${ }^{1}$ and Ma Guadalupe Aguilera-Arreola ${ }^{1 *}$
}

\begin{abstract}
Background: Lactobacillus jensenii, L. iners, L. crispatus and L. gasseri are the most frequently occurring lactobacilli in the vagina. However, the native species vary widely according to the studied population. The present study was performed to genetically determine the identity of Lactobacillus strains present in the vaginal discharge of healthy and bacterial vaginosis (BV) intermediate Mexican women.

Methods: In a prospective study, 31 strains preliminarily identified as Lactobacillus species were isolated from 21 samples collected from 105 non-pregnant Mexican women. The samples were classified into groups according to the Nugent score criteria proposed for detection of BV: normal (N), intermediate (I) and bacterial vaginosis (BV). We examined the isolates using culture-based methods as well as molecular analysis of the V1-V3 regions of the $16 \mathrm{~S}$ rRNA gene. Enterobacterial repetitive intergenic consensus (ERIC) sequence analysis was performed to reject clones.

Results: Clinical isolates (25/31) were classified into four groups based on sequencing and analysis of the 16S rRNA gene: L. acidophilus (14/25), L. reuteri (6/25), L. casei (4/25) and L. buchneri (1/25). The remaining six isolates were presumptively identified as Enterococcus species. Within the L. acidophilus group, L. gasseri was the most frequently isolated species, followed by L. jensenii and L. crispatus. L. fermentum, L. rhamnosus and L. brevis were also isolated, and were placed in the L. reuteri, L. casei and L. buchneri groups, respectively. ERIC profile analysis showed intraspecific variability amongst the L. gasseri and L. fermentum species.

Conclusions: These findings agree with previous studies showing that L. crispatus, L. gasseri and L. jensenii are consistently present in the healthy vaginal ecosystem. Additional species or phylotypes were detected in the vaginal microbiota of the non-pregnant Mexican (Hispanic-mestizo) population, and thus, these results further our understanding of vaginal lactobacilli colonisation and richness in this particular population.
\end{abstract}

Keywords: Lactobacilli, Mexican population, $16 \mathrm{~S}$ rRNA, Species identification, Vaginal microbiota, Bacterial vaginosis

\section{Background}

The vaginal ecosystem is dynamic and contains microbiota that are protective against invading pathogens, including those causing urinary tract infections and sexually transmitted infections (STIs). Lactobacilli are the best known bacteria of the normal vaginal microbiota. Their ability to produce lactic acid, $\mathrm{H}_{2} \mathrm{O}_{2}$ and bacteriocins

\footnotetext{
* Correspondence: lupita_aguilera@hotmail.com

'Laboratorio de Bacteriología Médica, Departamento de Microbiología, ENCB-IPN, México D.F., Mexico

Full list of author information is available at the end of the article
}

makes them prime candidates for the surveillance of vaginal health because these substances are unfavourable to many other bacterial species [1,2]. However, the presence of lactobacilli may not always be beneficial because certain lactobacilli do not contribute to vaginal wellbeing [1]. In general, a Lactobacillus-deficient condition, characterised by an overgrowth of anaerobes, is associated with the development of numerous infectious, such as bacterial vaginosis (BV) and aerobic vaginitis, and promotes the transmission of sexually transmitted diseases, including gonorrhoea, chlamydia, syphilis, trichomoniasis, human

\section{() Biomed Central}


immunodeficiency virus (HIV) and human papillomavirus (HPV) [3-5]. Lactobacillus jensenii, L. gasseri, L. iners and L. crispatus are the most frequently isolated Lactobacillus species from the vagina, although the native species and their relative abundance vary widely depending on the studied population $[3,6,7]$.

In Mexican women, an early phenotypic study suggested that $L$. acidophilus was the predominant species [8], whereas L. brevis, L. crispatus, L. fermentii and $L$. jensenii were identified in a more recent study using carbohydrate profiling [2]. Both of these studies used the vaginal discharge of healthy women. In contrast, when genetic approaches were used, $L$. acidophilus, L. iners, L. gasseri and L. delbrueckii were reported to be the most prevalent species in pregnant Mexican women [9]. To our knowledge, Lactobacillus species found in the vaginal discharge of healthy, non-pregnant Mexican women have not been studied using genetic approaches.

The unreliability of classical identification methods, which employed sugar fermentation and other phenotypic assays, previously hindered the identification of the predominant Lactobacillus species colonising the vagina. The inclusion of molecular tools for species identification is extremely important because previous studies have demonstrated that strains can be incorrectly classified when using the API $50 \mathrm{CHL}$ identification system alone [10,11]. Thus, microbiological and biochemical methods may be unreliable for the identification of lactic acid bacteria because of the low discriminatory power of the tests, and the intragenic diversity of Lactobacillus strains [12]. Therefore, the accurate characterisation of isolated strains may be achieved using a polyphasic approach, including a combination of classical culturedependent and culture-independent phenotyping methods, along with molecular procedures $[13,14]$. Randomly amplified polymorphic DNA (RAPD) analysis, ribotyping and intergenic spacer PCR (ITS-PCR) analysis are commonly employed molecular methods for strain identification $[10,12,15,16]$. However, $16 \mathrm{~S}$ ribosomal RNA sequence analysis is the most popular approach for classifying Lactobacillus strains. Analysis of 16S rRNA gene fragments is one the most powerful molecular tools for determining phylogenetic relationships among bacteria. In particular, 16S rRNA sequencing is useful in identifying bacteria that are difficult to classify by conventional methods.

Culture-dependent and culture-independent studies have found that $L$. jensenii, L. iners, $L$. crispatus and L. gasseri are the most common Lactobacillus species present in the vagina $[17,18]$. This study was performed to determine the genetic identity of Lactobacillus strains present in the vaginal discharge of healthy and bacterial vaginosis intermediate Mexican women.

\section{Methods}

\section{Sampling procedure and bacterial strains}

This study was performed with approval from the ethics committee of the National School of Biology Science (ENCB) at the National Polytechnic Institute (IPN), Mexico City. Written informed consent was obtained from all study participants. In total, 105 vaginal samples (vaginal exudates) to be used for culturing of Lactobacillus species were collected from 105 participating women at the ENCB. The women were between 18 and 65 years old. Exclusion criteria included pregnancy, antibiotic treatment within one month prior to sampling and sexual intercourse within three days prior to sampling. Demographic, behavioural and clinical analyses of the results from the survey of the enrolled participants have been published [19]. All samples were taken from the posterior zone of the fornix of the vagina using a sterile swab. The swab was placed in a tube containing Stuart broth (Difco, Detroit, MI, USA) and inoculated onto Man Rogosa Sharpe (MRS) agar plates (Oxoid, Basingstoke, Hampshire, England). A thin smear was examined by Gram staining and interpreted using Nugent's criteria. Three Lactobacillus reference strains from the ENCB culture collection were included, and characterised in parallel with the clinical isolates. Aeromonas caviae strain IIH147 was used as a positive control for the enterobacterial repetitive intergenic consensus (ERIC) sequence PCR assay.

\section{Sample groups}

The samples were classified into groups according to the Nugent score criteria proposed for normal (N), intermediate (I) and bacterial vaginosis (BV) [20]. If the Nugent score was normal, and if no other bacterial disease was found, the sample was categorised as healthy $(\mathrm{H})$. In contrast, if the Nugent score was intermediate or BV and other bacteria were present, the sample was classified as unhealthy (UH) (Figure 1). The scores were categorised as follows: $0-3$ was considered normal or negative for BV; 4-6 was considered intermediate; $\geq 7$ was considered indicative of BV.

\section{Strain isolation}

The swabs were inoculated onto MRS agar plates (Difco) and incubated at $37^{\circ} \mathrm{C}$ with partial $\mathrm{CO}_{2}$ tension for $48 \mathrm{~h}$. Growth on MRS was observed for 21 of the 105 analysed samples, and one or more colonies were selected and picked for each of the 21 samples. All colonies were examined by Gram staining and catalase and oxidase tests. A total of 31 strains that were phenotypically consistent with the Lactobacillus genus were chosen and preserved in $25 \%$ MRS glycerol at $-70^{\circ} \mathrm{C}$ until DNA extraction. 


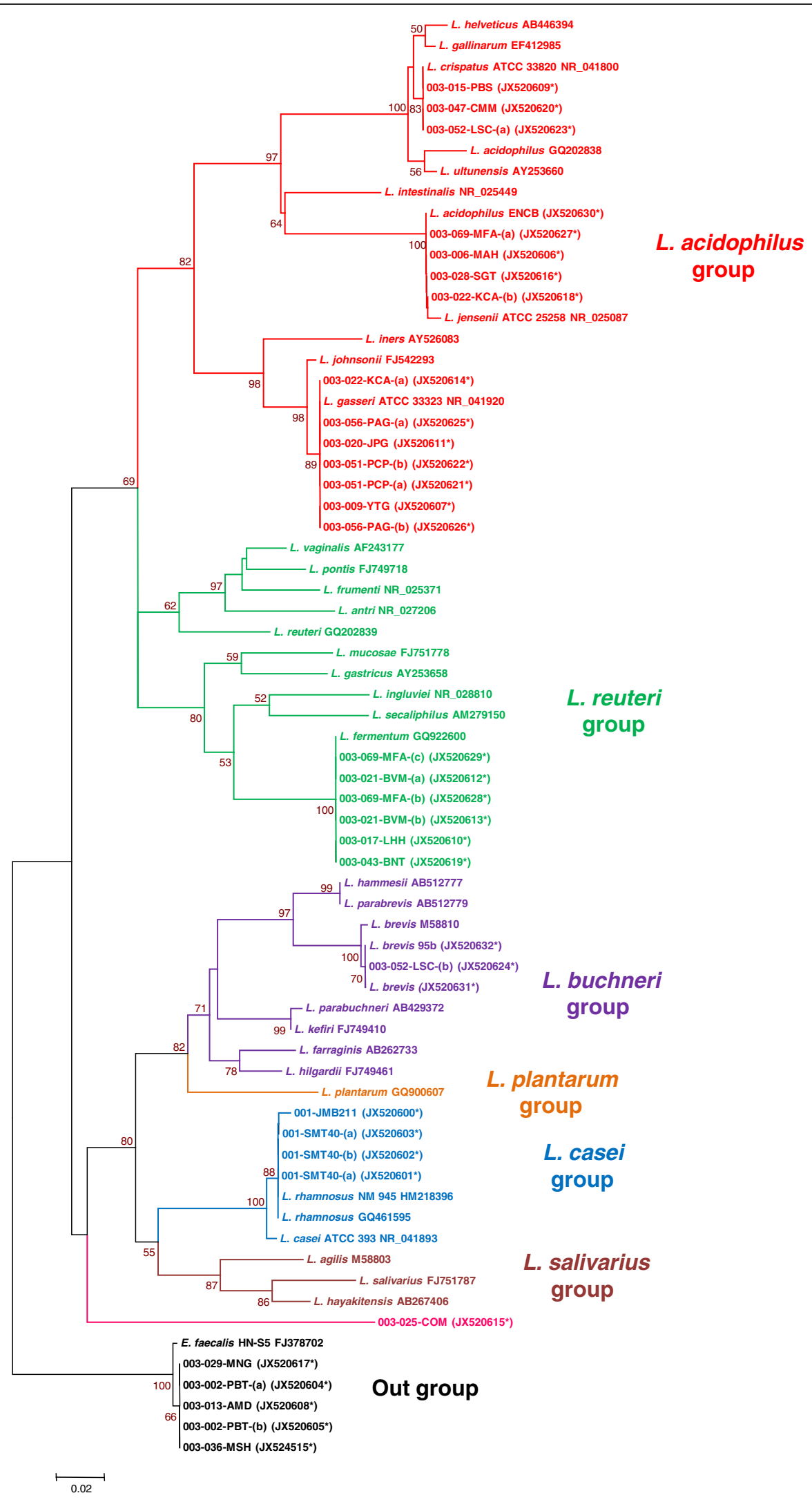

Figure 1 (See legend on next page.) 
(See figure on previous page.)

Figure 1 Dendrogram based on the 16S rRNA sequences of Lactobacillus strains identified in this study. The tree was generated using the neighbour-joining method. The branch lengths are proportional to the genetic distance, and the numbers shown at the branch points indicate the bootstrap values. The data set was subjected to 1,000 bootstrap replicates. The reference sequences were obtained from the GenBank database. Sequences from the clinical samples are indicated by an asterisk, and the sequence accession numbers are in parentheses (GenBank JX520600-JX520632).

\section{DNA extraction}

The isolated Lactobacillus strains were subcultured on MRS agar at $37^{\circ} \mathrm{C}$ for $48 \mathrm{~h}$. The bacterial colonies were added to $500 \mu \mathrm{L}$ of Tris-EDTA 50/20 (TE) buffer (pH 8) (Sigma-Aldrich, Saint Louis, MO, USA) and then mixed and centrifuged at $8000 \times g$ for $2 \mathrm{~min}$. The resulting supernatant was removed by decantation and discarded, and then $175 \mu \mathrm{L}$ of TE buffer and $10 \mu \mathrm{L}$ of RNase A (Sigma-Aldrich) were added to resuspend the cell pellet. The sample was then incubated at room temperature for $10 \mathrm{~min}$. The cells were lysed by the addition of $20 \mu \mathrm{L}$ of $10 \%$ sodium dodecyl sulphate (SDS) (Sigma-Aldrich) and $5 \mu \mathrm{L}$ of a proteinase $\mathrm{K}$ solution $(20 \mathrm{mg} / \mathrm{mL}$ ) (SigmaAldrich), followed by a $2 \mathrm{~h}$ incubation at $56^{\circ} \mathrm{C}$. Subsequently, $20 \mu \mathrm{L}$ of $5 \mathrm{M} \mathrm{NaCl}$ (Sigma-Aldrich) and $500 \mu \mathrm{L}$ of TE 10/1 ( $\mathrm{pH} \mathrm{8)}$ were added to the samples. The lysate was extracted once with $500 \mu \mathrm{L}$ of equilibrated phenol, which was mixed for $10 \mathrm{~min}$ and then centrifuged at $8000 \times g$ for $5 \mathrm{~min}$ at $4^{\circ} \mathrm{C}$. The supernatant was removed, and the aqueous phase was extracted once more with 500 $\mu \mathrm{L}$ of chloroform:isoamyl alcohol (24:1) and centrifuged at $10,000 \times g$ for $2 \mathrm{~min}$ at $4^{\circ} \mathrm{C}$. The supernatant was transferred to a sterile tube and $500 \mu \mathrm{L}$ of isopropanol was added. The mixture was centrifuged at $10,000 \times g$ for 2 min, after which the supernatant was removed. The pellet was washed with $10 \mu \mathrm{L}$ of $70 \%$ cold ethanol and centrifuged at $8,000 \times g$ for $2 \mathrm{~min}$. The pellets were airdried, and the DNA was resuspended in $100 \mu \mathrm{L}$ of DNasefree water. The 31 purified DNA samples were stored at $-20^{\circ} \mathrm{C}$ until use.

\section{ERIC-PCR analysis}

Because multiple colonies may have been selected from the same sample (Table 1), an ERIC-PCR analysis was performed on 14 isolates to identify if there were clones amongst the colonies. Genomic DNA was extracted as described above. The extracted DNA (100 ng) was used directly in a PCR reaction with previously described primers and conditions [21], conducted in a TGradient thermocycler (Biometra, Goettingen Germany). Twentyfive microlitre aliquots from each of the PCR amplifications were separated by electrophoresis for $2 \mathrm{~h}$ in $2 \%(\mathrm{~m} / \mathrm{v})$ agarose gels at $100 \mathrm{~V}$, and then visualised by ethidium bromide staining. The DNA molecular weight marker VI (0.15-2.1 kb) (Roche Applied Science, Indianapolis, IN, USA) was used to estimate product sizes. A negative reaction control was included, and the A. caviae IIH47 strain was used as a positive control for each ERICPCR run.

\section{Amplification of 165 rRNA genes}

DNA samples from the 31 isolates and three reference strains were subjected to PCR analysis. PCR was performed in a TGradient thermocycler (Biometra, Goettingen Germany), using primers that have been described previously [22]. The PCR amplifications were performed as follows: $2 \mu \mathrm{L}$ of the PCR template was used in a $50 \mu \mathrm{L}$ PCR mixture containing $1 \times$ PCR buffer with $2 \mathrm{mM} \mathrm{MgCl}_{2}$ (Invitrogen, São Paulo Brazil), $0.3 \mathrm{mM}$ of each dNTP (Invitrogen), $0.2 \mu \mathrm{M}$ of each primer, and 2.5 units of Taq DNA polymerase (Invitrogen). PCR amplification was conducted with the following temperature profile: an initial denaturation of $5 \mathrm{~min}$ at $94^{\circ} \mathrm{C}, 31$ cycles of $30 \mathrm{~s}$ at $94^{\circ} \mathrm{C}, 60 \mathrm{~s}$ at $55^{\circ} \mathrm{C}$, and $90 \mathrm{~s}$ at $72^{\circ} \mathrm{C}$, followed by $5 \mathrm{~min}$ at $72^{\circ} \mathrm{C}$. The PCR products were resolved by electrophoresis in a $1 \%(\mathrm{w} / \mathrm{v})$ agarose gel and visualised by ethidium bromide staining.

\section{DNA sequencing}

A total of 31 PCR products were purified using a PureLink Quick Gel Extraction Kit (Invitrogen, Carlsbad, CA, USA) according to the manufacturer's instructions. The products were directly sequenced on an ABI-PRISM 310 Genetic Analyzer (Applied Biosystems, Foster City, CA, USA) using the forward and reverse primers used for PCR, according to the manufacturer's instructions. Ambiguous and incorrectly called bases were manually corrected using Chromas Lite software, version 2.01 (Technelysium Pty Ltd.) and Seaview software version 4.3.3 [23]. To identify the isolates, the 31 sequences were compared to the V1-V3 regions of the lactobacilli $16 \mathrm{~S}$ rRNA gene sequences available in the GenBank DNA database using the BLAST algorithm (www.ncbi.nih. gov). Sequences from the top BLAST hits were downloaded for further phylogenetic comparison, and were from: L. iners AY526083, L. johnsonii FJ542293, L. gasseri AB517146, L. acidophilus GQ202838, L. ultunensis AY253660, L. gallinarum EF412985, L. helveticus AB446394, L. intestinalis NR_025449, L. ultunensis AY253660, L. gasseri ATCC 33323 NR_041920, L. jensenii ATCC 25258 NR_025087 and L. crispatus ATCC 33820 NR_041800 from the L. acidophilus group. L. mucosae FJ751778, L. gastricus AY253658, 
Table 1 Description of vaginal lactobacilli as determined by culture- and PCR-based identification

\begin{tabular}{|c|c|c|c|c|c|}
\hline Isolate & Age of participant $n=21$ & Lactobacillus group & Genetic ID & Nugent score & Co-infecting microorganism (s) \\
\hline \multicolumn{6}{|c|}{ Clinical isolates with Lactobacillus group $n=25$} \\
\hline 003-051-PCP-a† & 32 & acidophilus & L. gasseri & N & \\
\hline 003-051-PCP-b & & & L. gasseri & $\mathrm{N}$ & \\
\hline 003-056-PAG-ał & 22 & & L. gasseri & $\mathrm{N}$ & Uu \\
\hline 003-056-PAG-b & & & L. gasseri & $\mathrm{N}$ & Uu \\
\hline 003-020-JPG $†$ & 26 & & L. gasseri & $\mathrm{N}$ & \\
\hline 003-022-KCA-ał & 23 & & L. gasseri & । & $\mathrm{Uu}+\mathrm{Ca}$ \\
\hline 003-009-YTG† & 27 & & L. gasseri & N & \\
\hline 003-028-SGT‡ & 48 & & L. jensenii & $\mathrm{N}$ & Uu \\
\hline 003-006-MAH† & 22 & & L. jensenii & $\mathrm{N}$ & \\
\hline 003-022-KCA-b & & & L. jensenii & 1 & $\mathrm{Uu}+\mathrm{Ca}$ \\
\hline 003-069-MFA-a† & 20 & & L. jensenii & $\mathrm{N}$ & \\
\hline 003-052-LSC-at & 23 & & L. crispatus & $\mathrm{N}$ & \\
\hline 003-047-CMM† & 36 & & L. crispatus & $\mathrm{N}$ & \\
\hline 003-015-PBS† & 22 & & L. crispatus & $\mathrm{N}$ & \\
\hline 003-021-BVM-ał & 21 & reuteri & L. fermentum & $\mathrm{N}$ & Uu \\
\hline 003-021-BVM-b & & & L. fermentum & $\mathrm{N}$ & Uu \\
\hline 003-069-MFA-b & & & L. fermentum & $\mathrm{N}$ & \\
\hline 003-069-MFA-c & & & L. fermentum & $\mathrm{N}$ & \\
\hline 003-017-LHH† & 50 & & L. fermentum & $\mathrm{N}$ & \\
\hline 003-043-BNT‡ & 26 & & L. fermentum & $\mathrm{N}$ & GV \\
\hline 001-JMB211‡ & 38 & casei & L. rhamnosus & $\mathrm{N}$ & $\mathrm{Ca}$ \\
\hline 001-SMT40-a†¥ & 49 & & L. rhamnosus & $\mathrm{N}$ & \\
\hline 001-SMT40-b¥ & & & L. rhamnosus & $\mathrm{N}$ & \\
\hline 001-SMT40-c & & & L. rhamnosus & $\mathrm{N}$ & \\
\hline 003-052-LSC-b & & buchneri & L. brevis & $\mathrm{N}$ & \\
\hline \multicolumn{6}{|c|}{ Strains without Lactobacillus group, $n=6$} \\
\hline 003-025-COM† & 38 & & L sp. & $\mathrm{N}$ & \\
\hline 003-002-PBT-ał & 25 & & E. faecalis* & । & $\mathrm{Uu}+\mathrm{Ct}$ \\
\hline 003-002-PBT-b & & & E. faecalis* & । & $\mathrm{Uu}+\mathrm{Ct}$ \\
\hline 003-036-MSH† & 49 & & E. faecalis* & ND & \\
\hline 003-029-MNG $\neq$ & 45 & & E. faecalis* & $\mathrm{N}$ & Uu \\
\hline 003-013-AMD $\ddagger$ & 28 & & E. faecalis* & $\mathrm{N}$ & Uu \\
\hline \multicolumn{6}{|c|}{ Reference strains, $n=3$} \\
\hline L. brevis $95 \mathrm{~b}$ & NA & buchneri & L. brevis & NA & NA \\
\hline L. brevis 95 a & NA & & L. brevis & NA & NA \\
\hline L. acidophilus ENCB & & acidophilus & L. jensenii & NA & NA \\
\hline
\end{tabular}

Normal $=0-3(\mathrm{~N})$, intermediate $=4-6(\mathrm{l}), \mathrm{Ct}=$ Chlamydia trachomatis, $\mathrm{Uu}=$ Ureaplasma urealyticum, $\mathrm{Gv}=$ Gardnerella vaginalis, $\mathrm{Ca}=\mathrm{Candida}$ spp., $\mathrm{NA}=$ not applicable, ND not determined. ${ }^{*}$ presumptive identification. ${ }^{\dagger}$ healthy, ${ }^{\mp}$ unhealthy. ${ }^{*} 001-$ SMT40-a and 001-SMT-b are clones. 001-SMT40, 003-017-LHH and 003-036 -MSH samples were from postmenopausal women.

L. secaliphilus AM279150, L. fermentum GQ922600, L. ingluviei NR_028810, L. reuteri GQ202839, L. pontis FJ749718, L. antri NR_027206, L. vaginalis AF243177 and L. frumenti NR_025371 from the L. reuteri group. L. kefiri FJ749410, L. parabuchneri AB429372, L. hilgardii
FJ749461, L. farraginis AB262733, L. hammesii AB512777, L. parabrevis AB512779 and L. brevis M58810 from the L. reuteri group. L. plantarum GQ900607 from the $L$. plantarum group. L. agilis M58803, L. salivarius FJ751787 and L. hayakitensis AB267406 from the L. salivarius 
group. L. casei ATCC 393 NR_041893 and L. rhamnosus NM-945 HM218396 from the L. casei group. E. faecalis HNS5 FJ378702 was also included.

A multiple sequence alignment was performed using the program Clustal X, version 2.0 [24], and the resulting alignment was edited using SeaView [25]. A phylogenetic tree was constructed based on the sequence distances using the neighbour-joining (NJ) algorithm with the Tamura-Nei substitution model. The phylogenetic analyses were performed using Mega 4 [26]. The stability or accuracy of the inferred topology was assessed via a bootstrap analysis of 1,000 replicates. The identities of the sequences were determined based on the highest percentage (a minimum of 97\%) of the total nucleotide match with sequences from GenBank [27,28].

\section{Results}

In total, 31 isolates from 21 vaginal discharge samples from healthy $(\mathrm{H} ; \mathrm{n}=12)$ and BV intermediate (unhealthy) $(\mathrm{UH} ; \mathrm{n}=9)$ non-pregnant Mexican participants were phenotypically identified. All isolates were Gram-positive, non-motile, non-spore forming, catalase-negative, short or large rods, with morphology characteristic of Lactobacillus when grown on MRS medium. The quality and purity of genomic DNA from the 31 isolates and the three reference strains was sufficient to amplify the $16 \mathrm{~S}$ rRNA gene, and partial 16S rRNA gene sequences were obtained for all samples. Because the first 510 bp of this gene include the variable regions V1-V3, which provide sufficient information for sequence analysis, only this region was considered in the in vitro analyses. To identify the isolates, a comparison of the sequences obtained in this study with those deposited in the GenBank database was performed using DNA sequence alignment and bioinformatics analysis. Sequences identified as Lactobacillus, specific species related to the vaginal habitat, and named and verified type strains deposited in the database at the time of retrieval were used in the analysis. Figure 1 shows the phylogenetic tree obtained from the sequence alignment.

The sequences of the 25 (25/31) Lactobacillus isolates were classified into the four groups of lactobacilli described by Ljungh and Wadström [29] as follows: 14 isolates $(14 / 25)$ were classed as L. acidophilus, six isolates $(6 / 25)$ were $L$. reuteri, four isolates $(4 / 25)$ were $L$. casei and one isolate $(1 / 25)$ was classed as L. buchneri. These results were similar to what has been reported in the literature. Surprisingly, five strains (5/31) were presumptively identified as E. faecalis by molecular characterisation, and one strain $(1 / 31)$ could not be identified at the species level and was not clustered within any of the Lactobacillus groups proposed by Ljungh and Wadström. Using Nugent's criteria, 26 strains were isolated from vaginal discharge that was scored as normal (26/31), four were obtained from samples scored as intermediate (4/31) and one sample was not evaluated using Nugent's criteria (1/31).

Among the participants from which lactobacilli were isolated ( $\mathrm{n}=16), 13$ were colonised by one Lactobacillus species, and three individuals were colonised by two different lactobacilli (Table 1). The remaining five samples contained Enterococcus and Lactobacillus species. Because more than one colony could have been chosen from the same sample, an ERIC-PCR analysis was performed $(n=14)$. The analysis showed that only two isolates from one sample were clones (SMT40). In contrast, the profile analysis indicated the occurrence of intraspecific variability among the $L$. gasseri and $L$. fermentum species. For example, different ERIC-PCR profiles between isolates from the same sample were obtained, including PCP-a vs. PCP-b, PAG-a vs. PAG-b, BVM-a vs. BVM-b, and MFA-b vs. MFA-c, and between strains isolated from different samples, including PCP ( $a$ or b) vs. PAG ( $a$ or b) and BVM ( $a$ or b) vs. MFA ( $b$ or c).

Because other microorganisms were identified in a previous study that had 105 samples [17], the incidence of co-infection could be analysed. Co-infection with lactobacilli and Chlamydia trachomatis, Ureaplasma urealyticum, Gardnerella vaginalis and Candida albicans was observed. L. gasseri was the most frequently isolated species $(\mathrm{n}=7 ; \mathrm{H}=4, \mathrm{UH}=3)$, followed by L. fermentum $(\mathrm{n}=6 ; \mathrm{H}=3, \mathrm{UH}=3)$, L. jensenii $(\mathrm{n}=4 ; \mathrm{H}=2, \mathrm{UH}=2)$, L. crispatus $(\mathrm{n}=3 ; \mathrm{H}=3, \mathrm{UH}=0)$, L. rhamnosum $(\mathrm{n}=3$; $\mathrm{H}=2, \mathrm{UH}=1)$ and L. brevis $(\mathrm{n}=1 ; \mathrm{H}=1, \mathrm{UH}=0)$. Because of the low number of isolates, it was not possible to conclusively determine whether a particular species was associated with a healthy or unhealthy status. However, in general, L. gasseri (4/7) and L. crispatus (3/3) were more frequently detected in the normal samples than in the "unhealthy" group (Table 1). When the interpretation of the smears according to Nugent's criteria was considered, the Lactobacillus isolates were classified into only two of the three possible groups (normal and intermediate); accordingly, Lactobacillus was not isolated from the samples with BV, although one isolate was co-isolated with G. vaginalis (Figure 2).

\section{Discussion}

Lactobacilli are an important part of the vaginal microbiota. The presence of lactic acid, $\mathrm{H}_{2} \mathrm{O}_{2}$ and other byproducts of these bacteria are beneficial in controlling the overgrowth of potentially pathogenic bacteria (i.e., bacterial vaginosis). For example, Antonio et al. [30] reported that women who were not colonised with $\mathrm{H}_{2} \mathrm{O}_{2}$ producing lactobacilli, such as $L$. crispatus, $L$. iners, $L$. jensenii, L. gasseri and L. vaginalis, were 15 times more likely to have bacterial vaginosis than woman who were colonised by these strains. Despite their importance to women's health, vaginal lactobacilli have not been 


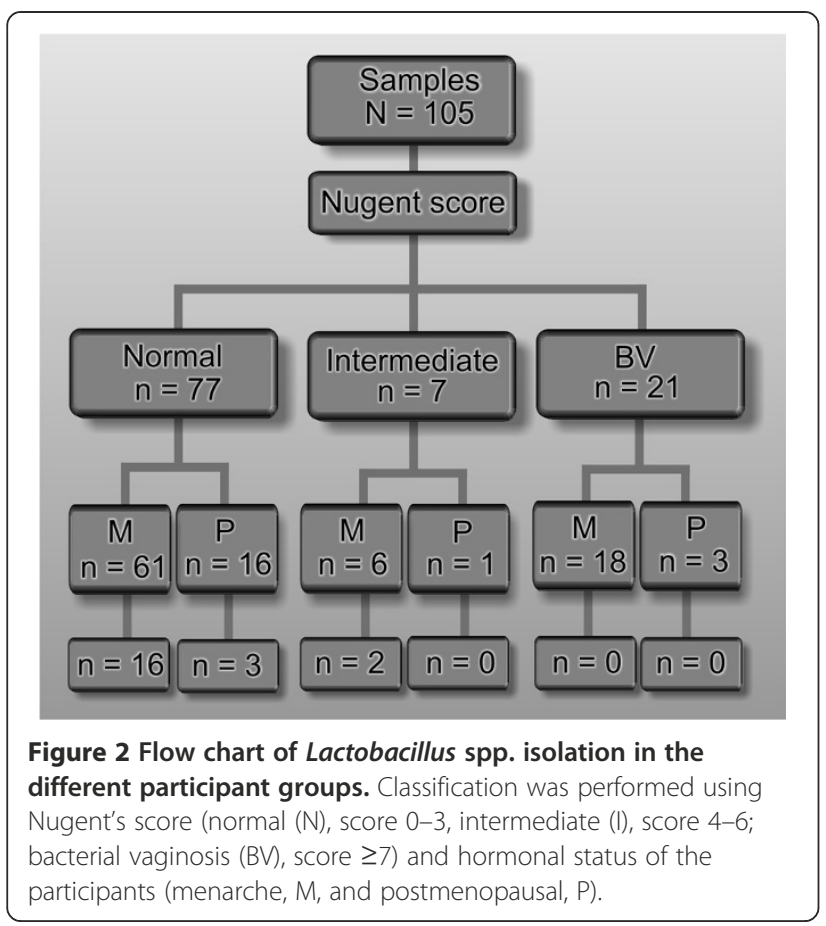

extensively studied in the Mexican population. Historically, L. acidophilus was considered to be the dominant species in the human vagina. It is now known that the group of organisms previously known as L. acidophilus is highly heterogeneous, and includes at least six separate species [29,31]. In addition, it has been shown that the majority of vaginal Lactobacillus strains from women of geographically separated countries belong to the four species $L$. iners, $L$. crispatus, $L$. gasseri and $L$. jensenii, indicating a high degree of species consistency in vaginal lactobacilli among women worldwide [32]. The identity of the vaginal lactobacilli in Mexican women has not been well-studied, and the majority of published papers have used phenotypic approaches. Therefore, we based our approach on $16 \mathrm{~S}$ rRNA gene sequencing and ERIC-PCR analysis.

The isolation of lactobacilli in the present study was performed using a general selective medium, and relatively few strains were isolated. This result is consistent with other reports, which indicate that lactobacilli may go undetected in the laboratory because their growth requires unique media and an extended incubation time [31]. In 2001, Angeles-Lopez et al. [2] reported the isolation of lactobacilli in only 87 of 156 samples inoculated on MRS agar. In addition, it has been reported that $L$. iners, a species belonging to the $L$. acidophilus group, does not grow on MRS agar. Therefore, in the present study and other studies using MRS, this species was not evaluated and has been misidentified [33]. Other authors have noted that even after recovery, strain misidentification can occur because the strains morphologically resemble those of other genera, including Corynebacterium, Clostridium and Streptococcus [31]. Given the difficulty in isolating these bacteria, and the possibility that they may often be misidentified, culture-independent genetic approaches are now preferred over culturedependent methods [34]. Moreover, several types of vaginal microbiota exist in healthy women. Although Lactobacillus is often the predominant genus, the vaginal microbiota also includes a diverse assemblage of anaerobic microorganisms, which likely occur within the mestizo and Mexican populations.

When strains can be isolated, their identification can be aided by molecular techniques to distinguish between closely related species within the Lactobacillus genus, which can be impossible by phenotypic methods alone. Although a large number of molecular methodologies are currently available to study these bacteria, some of the techniques, such as PCR, denaturing gradient gel electrophoresis (DGGE) and thermal gradient gel electrophoresis (TGGE) [9,34-37], still require improvement, especially with regard to the sensitivity, cost and quantitative power. Among all available molecular techniques, $16 \mathrm{~S}$ rRNA sequencing analysis has been accepted as the most reliable method. Therefore, this method was used in the present work to provide the first genetic identification of the indigenous microbiota of the vaginal cavity of non-pregnant Mexican women.

The majority of the species detected in the Mexican population in the present study belong to the L. acidophilus group, although strains of the L. reuteri, L. casei and L. buchneri groups have also been identified [29]. The majority of the lactobacilli found in the vaginal communities were phylogenetically related to L. gasseri, L. fermentum, $L$. rhamnosus, $L$. jensenii, $L$. crispatus, $L$. fornicalis or $L$. brevis, which is at least partially consistent with previous reports on the species distribution in other countries $[6,17,32,38]$. However, other lactobacilli species have been described at a lower frequency, including $L$. vaginalis, $L$. fermentum, L. mucosae, L. paracasei and L. rhamnosus, in reports that have indicated the variability among women of specific regions $[6,32,39,40]$. This trend is consistent with our results, in which $L$. fermentum, L. rhamnosus and $L$. brevis were also identified. These findings are not surprising since it has become increasingly apparent in recent years that ethnicity can affect the number and type of organisms present in the vaginal cavity $[17,18,37]$.

A previous study published by Hernández-Rodríguez et al. [9] did not identify L. crispatus using a culture-independent method (DGGE-PCR) in samples from pregnant Mexican women, which is in contrast with our study, in which this species was isolated, albeit at a low frequency $[17,18,38,40]$.

The absence of $L$. iners isolates in our study is significant because it is one of the dominant species reported 
worldwide. The lack of detection of $L$. iners could be attributed to the limitations of the methodology used in the current analysis, and must be confirmed in future studies without these limitations (e.g. DGGE of PCRamplified 16S rRNA fragments can overcome the limitation of traditional cultivation techniques to retrieve the vaginal econiche diversity).

Correct species identification is dependent on the reliability of the reference strains and accuracy of the database used. Because many reference strains were previously characterised using non-genetic methods, it is possible that they were misidentified and, thus, the reference strains themselves may be unreliable [32]. For example, the reference strain previously identified as $L$. acidophilus should be re-labelled as $L$. jensenii based on the genetic data obtained in the present study (Table 1). Both species belong to the L. acidophilus group and are genetically related.

The majority of the strains isolated in this study were identified as species of the $L$. acidophilus group. Because Lactobacillus species are considered to be critical for protection against pathogens in the female genital tract, this set of strains could be useful in future studies on probiotic properties [29], especially given the potential differences in the protective capabilities of vaginal Lactobacillus species.

The intraspecific variability among the $L$. gasseri and L. fermentum species, detected by ERIC-PCR analysis, concurs with a previous report by Stephensen et al. [41]. This previous study showed that ERIC-PCR analysis was capable of typing Lactobacillus isolates at the strain level.

The main limitations of this study were the small sample size and problems associated with culturedependent methods. In addition, because of the small number of isolates, it was not possible to correlate the observed species with a healthy or unhealthy (BV) status. Our results should be corroborated using a larger cohort and culture-independent methods (DGGE or sequencing of cloned 16S rRNA molecules) to describe the relative abundance of the species described herein. Despite these limitations, the results of this study concur with previously published findings showing that $L$. crispatus, $L$. gasser $i$ and $L$. jensenii are consistently present in the healthy vaginal ecosystem, and provide additional information regarding the Mexican (Hispanic-mestizo) non-pregnant population $[6,17,32,38,40,42]$. Additional species or phylotypes not common in other countries were found in this study, which furthers our understanding of vaginal colonisation by lactobacilli, and the Lactobacillus species diversity in vaginal communities in the mestizo population.

\section{Conclusions}

Accurate phenotypic identification of species of the genus Lactobacillus is difficult. The use of molecular techniques in combination with culture-based methods adds greatly to our understanding of the normal microbiota of a particular environment. The Mexican (Hispanic-mestizo) non-pregnant population is colonized mainly by $L$. acidophilus group lactobacilli. The majority of the lactobacilli identified in the Mexican vaginal communities were $L$. gasseri, $L$. fermentum, $L$. rhamnosus, L. jensenii, L. crispatus and L. brevis. Because the dominant Lactobacillus species may differ depending on race or geography, the ability to identify lactobacilli at the species level should enable us to better understand the roles of the various Lactobacillus species. Culture-independent techniques must be used in future analyses to overcome the difficulty in isolating these bacteria and to prevent misidentification. Molecular methods can also provide a wider description of microbial communities, and measure prevalence, diversity and abundance of vaginal microbiota, which also includes a diverse assemblage of anaerobic microorganisms or more fastidious lactic acid-producing bacteria.

\section{Competing interests}

The authors declare that they have no competing interests.

\section{Authors' contributions}

MDMP carried out the molecular genetic studies, participated in the sequence alignment and helped to draft the manuscript. GCE helped to draft the manuscript and participated in the data analysis. MGAA conceived of the study, participated in its design and coordination, assessed the data and drafted the manuscript. All authors read and approved the final manuscript.

\section{Acknowledgments}

This study was funded by the research and graduate secretary (SIP) of the National Polytechnic Institute (IPN) (SIP 20100629 and 20110246) and CONACYT 118797. The SIP-IPN or CONACYT were not involved in the development of the study design, the collection, analysis, or interpretation of the data, in the writing of the report, or in the decision to submit the paper for publication.

\section{Author details}

${ }^{1}$ Laboratorio de Bacteriología Médica, Departamento de Microbiología, ENCB-IPN, México D.F., Mexico. ${ }^{2}$ Laboratorio de Recursos Genéticos Microbianos, Centro Nacional de Recursos Genéticos, INIFAP, Tepatitlán de Morelos, Jalisco, Mexico.

Received: 25 August 2012 Accepted: 19 April 2013

Published: 26 April 2013

\section{References}

1. Ma B, Forney LJ, Ravel J: Vaginal microbiome: rethinking health and disease. Annu Rev Microbiol 2012, 66:371-389.

2. Angeles-López M, García-Cano E, Aquino SC: Hydrogen peroxide production and resistance to nonoxinol-9 in Lactobacillus spp. isolated from the vagina of reproductive age women. Rev Latinoam Microbiol 2001, 43:171-176.

3. Antonio MA, Hawes SE, Hillier SL: The identification of vaginal Lactobacillus species and the demographic and microbiologic characteristics of women colonized by these species. J Infect Dis 1999, 180:1950-1956.

4. Turovskiy $Y$, Sutyak Noll K, Chikindas ML: The aetiology of bacterial vaginosis. J Appl Microbiol 2011, 110:1105-1128.

5. Gillet $E$, Meys JF, Verstraelen $H$, Bosire C, De Sutter P, Temmerman M, Broeck DV: Bacterial vaginosis is associated with uterine cervical human papillomavirus infection: a meta-analysis. BMC Infect Dis 2011, 11:10 [Published online]. 
6. Zhou X, Brown CJ, Abdo Z, Davis CC, Hansmann MA, Joyce P, Foster JA, Forney $\sqcup$ : Differences in the composition of vaginal microbial communities found in healthy Caucasian and black women. ISME J 2007, 1:121-133.

7. Vásquez A, Jakobsson T, Ahrné S, Forsum U, Molin G: Vaginal Lactobacillus flora of healthy Swedish women. J Clin Microbiol 2002, 40:2746-2749.

8. Pérez-Miravete A: Studies on the vaginal flora IX Classification of Lactobacilli of vaginal origin. Rev Latinoam Microbiol Parasitol 1967, 9:11-14.

9. Hernández-Rodríguez C, Romero-González R, Albani-Campanario M, Figueroa-Damián R, Meraz-Cruz N, Hernández-Guerrero C: Vaginal microbiota of healthy pregnant Mexican women is constituted by four Lactobacillus species and several vaginosis-associated bacteria. Infect Dis Obstet Gynecol 2011, 2011:851485.

10. Nigatu A: Evaluation of numerical analyses of RAPD and API $50 \mathrm{CH}$ patterns to differentiate Lactobacillus plantarum, L. fermentum, L. rhamnosus, L. sake, L. parabuchneri, L. gallinarum, L. casei, Weissella minor and related taxa isolated from kocho and tef. J Appl Microbiol 2000, 89:969-978.

11. Boyd MA, Antonio MA, Hillier SL: Comparison of API $50 \mathrm{CH}$ strips to whole-chromosomal DNA probes for identification of Lactobacillus species. J Clin Microbiol 2005, 43:5309-5311.

12. Srinivasan S, Fredricks DN: The human vaginal bacterial biota and bacterial vaginosis. Interdiscip Perspect Infect Dis 2008, 2008:750479.

13. Verhelst $R$, Verstraelen $H$, Claeys $G$, Verschraegen $G$, Van Simaey $L$, De Ganck C, De Backer E, Temmerman M, Vaneechoutte M: Comparison between Gram stain and culture for the characterization of vaginal microflora: definition of a distinct grade that resembles grade I microflora and revised categorization of grade I microflora. BMC Microbiol 2005, 14(5):61

14. Santiago GL, Cools $P$, Verstraelen $H$, Trog M, Missine $G$, El Aila N, Verhelst $R$, Tency I, Claeys G, Temmerman M, Vaneechoutte M: Longitudinal study of the dynamics of vaginal microflora during two consecutive menstrual cycles. PLoS One 2011, 6(11):e28180

15. Tannock GW, Tilsala-Timisjarvi A, Rodtong S, Ng J, Munro K, Alatossava T: Identification of Lactobacillus isolates from the gastrointestinal tract, silage, and yoghurt by 16S-23S rRNA gene intergenic spacer region sequence comparisons. Appl Environ Microbiol 1999, 65:4264-4267.

16. O'Sullivan DJ: Methods for analysis of the intestinal microflora. Curr Issues Intest Microbiol 2000, 1:39-50.

17. Ravel J, Gajer P, Abdo Z, Schneider GM, Koenig SS, McCulle SL, Karlebach S, Gorle R, Russell J, Tacket CO, Brotman RM, Davis CC, Ault K, Peralta L, Forney $\cup$ : Vaginal microbiome of reproductive-age women. Proc Natl Acad Sci USA 2011, 108:4680-4687.

18. Verstraelen $H$, Verhelst $R$, Claeys $G$, De Backer $E$, Temmerman M, Vaneechoutte M: Longitudinal analysis of the vaginal microflora in pregnancy suggests that L. crispatus promotes the stability of the normal vaginal microflora and that $\mathrm{L}$. gasseri and/or $\mathrm{L}$. iners are more conducive to the occurrence of abnormal vaginal microflora. BMC Microbiol 2009, 9:116.

19. Hernández-Martínez F, Hernández-García JA, Martínez-Peña MD, MuñizBecerril BL, Hernández-Cortez C, Castro-Escarpulli G, Aguilera-Arreola MG: Aetiology and frecuency of cervico-vaginal infections among Mexican women. AJMR 2013, 7:27-34

20. Nugent RP, Krohn MA, Hillier SL: Reliability of diagnosing bacterial vaginosis is improved by a standardized method of gram stain interpretation. J Clin Microbiol 1991, 29:297-301.

21. Vila J, Marcos MA, Jimenez de Anta MT: A comparative study of different PCR-based DNA fingerprinting techniques for typing of the Acinetobacter calcoaceticus-A. baumannii complex. J Med Microbiol 1996, 44:482-489.

22. Borrell N, Acinas SG, Figueras MJ, Martínez-Murcia AJ: Identification of Aeromonas clinical isolates by restriction fragment length polymorphism of PCR-amplified 16S rRNA genes. J Clin Microbiol 1997, 45:1671-1674.

23. Gouy M, Guindon S, Gascuel O: SeaView version 4: a multiplatform graphical user interface for sequence alignment and phylogenetic tree building. Mol Biol Evol 2010, 27:221-224.

24. Larkin MA, Blackshields G, Brown NP, Chenna R, McGettigan PA, McWilliam H, Valentin F, Wallace IM, Wilm A, López R, Thompson JD, Gibson FJ, Higgins DG: Clustal W and clustal X version 2 0. Bioinformatics 2007, 23:2947-2948.

25. Galtier N, Gouy M, Gautier C: SEAVIEW and PHYLO_WIN: two graphic tools for sequence alignment and molecular phylogeny. Comput Applic BiosCi 1996, 12:543-548.
26. Tamura K, Dudley J, Nei M, Kumar S: MEGA4: Molecular Evolutionary Genetics Analysis (MEGA) software version 4 0. Mol Biol Evol 2007, 24:1596-1599.

27. Rosselló-Mora R, Amann R: The species concept for prokaryotes. FEMS Microbiol Rev 2001, 25:39-67.

28. Morgulis A, Coulouris G, Raytselis Y, Madden $T L$, Agarwala R, Schäffer AA: Database indexing for production megablast searches. Bioinformatics 2008, 24:1757-1764.

29. Ljungh A, Wadström T: Lactobacillus Molecular Biology: From Genomics to Probiotics. Norfolk, UK: Caister Academic Press; 2009.

30. Antonio MA, Rabe LK, Hillier SL: Colonization of the rectum by Lactobacillus species and decreased risk of bacterial vaginosis. J Infect Dis 2005, 192:394-398.

31. Slover CM, Danzinger L: Lactobacillus: a review. Clin Microbio/ News/2008, 30:23-27.

32. Pavlova SI, Kilic AO, Kilic SS, So JS, Nader-Macias ME, Simoes JA, Tao L: Genetic diversity of vaginal lactobacilli from women in different countries based on 16S rRNA gene sequences. J Appl Microbio/ 2002, 92:451-459.

33. Falsen E, Pascual C, Sjödén B, Ohlén M, Collins MD: Phenotypic and phylogenetic characterization of a novel Lactobacillus species from human sources: description of Lactobacillus iners sp nov. Int J Syst Bacteriol 1999, 49:217-221.

34. Petricevic L, Domig KJ, Nierscher FJ, Krondorfer I, Janitschek C, Kneifel W, Kiss H: Characterisation of the oral, vaginal and rectal Lactobacillus flora in healthy pregnant and postmenopausal women. Eur J Obstet Gynecol Reprod Biol 2012, 160:93-99.

35. Dimitonova SP, Bakalov BV, Aleksandrova-Georgieva RN, Danova ST: Phenotypic and molecular identification of lactobacilli isolated from vaginal secretions. J Microbiol Immunol Infect 2008, 41:469-477.

36. Anukam KC, Osazuwa EO, Ahonkhai I, Reid G: Lactobacillus vaginal microbiota of women attending a reproductive health care service in Benin city, Nigeria. Sex Transm Dis 2006, 33:59-62.

37. Jespers V, Menten J, Smet H, Poradosú S, Abdellati S, Verhelst R, Hardy L, Buvé A, Crucitti T: Quantification of bacterial species of the vaginal microbiome in different groups of women, using nucleic acid amplification tests. BMC Microbio/ 2012, 12:83.

38. Zhou X, Hansmann MA, Davis CC, Suzuki H, Brown CJ, Schütte U, Pierson $J D$, Forney $\sqcup$ : The vaginal bacterial communities of Japanese women resemble those of women in other racial groups. FEMS Immunol Med Microbiol 2010, 58:169-181

39. De Backer E, Verhelst R, Verstraelen H, Alqumber MA, Burton JP, Tagg JR, Temmerman M, Vaneechoutte M: Quantitative determination by real-time PCR of four vaginal Lactobacillus species, Gardnerella vaginalis and Atopobium vaginae indicates an inverse relationship between $L$. gasseri and L. iners. BMC Microbio/ 2007, 7:115.

40. Fredricks DN, Fiedler TL, Marrazzo JM: Molecular identification of bacteria associated with bacterial vaginosis. N Engl J Med 2005, 353:1899-1911.

41. Stephenson DP, Moore RJ, Allison GE: Comparison and utilization of repetitive-blement PCR techniques for typing Lactobacillus isolates from the chicken gastrointestinal tract. Appl Environ Microbiol 2009, 75:6764-6776.

42. Spear GT, Gilbert D, Landay AL, Zariffard R, French AL, Patel P, Gillevet PM: Pyrosequencing of the genital microbiotas of HIV-seropositive and -seronegative women reveals Lactobacillus iners as the predominant Lactobacillus species. App/ Environ Microbiol 2011, 77:378-381.

doi:10.1186/1471-2334-13-189

Cite this article as: Martínez-Peña et al.: Lactobacillus species isolated from vaginal secretions of healthy and bacterial vaginosis-intermediate Mexican women: a prospective study. BMC Infectious Diseases 2013 13:189. 